\title{
Analysis of scientific collaboration in Chinese psychiatry research
}

\author{
Ying $\mathrm{Wu}^{1^{*}}$ and Xing Jin ${ }^{2}$
}

\begin{abstract}
Background: In recent decades, China has changed profoundly, becoming the country with the world's second-largest economy. The proportion of the Chinese population suffering from mental disorder has grown in parallel with the rapid economic development, as social stresses have increased. The aim of this study is to shed light on the status of collaborations in the Chinese psychiatry field, of which there is currently limited research.

Methods: We sampled 16,224 publications (2003-2012) from 10 core psychiatry journals from Chinese National Knowledge Infrastructure (CNKI) and WanFang Database. We used various social network analysis (SNA) methods such as centrality analysis, and Core-Periphery analysis to study collaboration. We also used hierarchical clustering analysis in this study.

Results: From 2003-2012, there were increasing collaborations at the level of authors, institutions and regions in the Chinese psychiatry field. Geographically, these collaborations were distributed unevenly. The 100 most prolific authors and institutions and 32 regions were used to construct the collaboration map, from which we detected the core author, institution and region. Collaborative behavior was affected by economic development.

Conclusion: We should encourage collaborative behavior in the Chinese psychiatry field, as this facilitates knowledge distribution, resource sharing and information acquisition. Collaboration has also helped the field narrow its current research focus, providing further evidence to inform policymakers to fund research in order to tackle the increase in mental disorder facing modern China.
\end{abstract}

Keywords: Psychiatry, Scientific collaboration, China

\section{Background}

China is now the world's second largest economy country, having seen profound changes in recent decades. The proportion of the Chinese population suffering from mental disorder has grown in parallel with the rapid economic development, as social stresses have increased. According to recent data from the World Health Organization (WHO), the burden of mental disorder is highest, surpassing that of cardiovascular disease, respiratory system disease and malignancy [1]. As it drew the attention of Chinese researchers studying psychiatry, the prevention and control of mental disorder became a huge challenge for them to overcome. Since this field permeates several arenas of biomedicine, no single individual is trained to perform all specialty tasks. Thus,

\footnotetext{
* Correspondence: wuguaiguai9896@126.com

${ }^{1}$ School of Humanities and Social Sciences, Shanxi Medical University, No. 56,

South Xinjian Road, Taiyuan, China

Full list of author information is available at the end of the article
}

scientific collaboration, which improves communication, facilitates sharing of expertise, and provides opportunities for the emergence of new scientific ideas, is indispensable for the growth of the field of psychiatry in modern China.

There has been increased collaboration within and between different scientific fields over the last decade. Co-authorship is a frequently used and reliable measure of research collaboration [2]. In 2001, American researcher Newman used Social network analysis (SNA) to study on the structure of scientific collaboration networks in fields such as biomedicine, physics and computer science [3-5]. German scholar Kretschmer applied the method of science, information metrology and psychology in scientific collaboration skillfully and acquired a series of achievements by using SNA [6, 7]. SNA has been used to study collaboration in bibliographic co-authorship networks $[8,9]$. A social network 
is defined as a set of social entities, such as people, organizations, and countries, with some pattern of relationship between them [10]. These networks are usually modeled by graphs, where nodes represent the social entities and lines represent the ties established between them. The underlying structure of such networks is the object of study of SNA.

There has, however, been a lack of publications on scientific collaboration within the Chinese psychiatry field. Therefore, this study was designed to measure the activities of this field at the micro level (authors), meso level (institutions) and macro level (regions).

\section{Methods}

We collected our data from 10 major journals in China National Knowledge Infrastructure (CNKI) and WanFang Database (2003 to 2012). Together, CNKI and WanFang Database cover the majority of journals from China. Each bibliographic record includes information such as the title, author names, abstract and key words. The 10 major journals include: (1) Journal of Clinical Mental Psychiatry, (2) Chinese Journal of Nervous and Mental Disease, (3) Journal of Neuroscience, (4) Journal of Clinical Psychosomatic Diseases, (5) Journal of Psychiatry, (6) Shang Hai Archives of Psychiatry, (7) Si Chuan Mental Health, (8) Chinese Journal of Psychiatry, (9) Journal of Neurology and Neurorehabilitation, and (10) Journal of International Psychiatry (Table 1). We believe that these 10 publications are sufficient to determine the structure of collaboration in the field of Chinese psychiatry research.

Centrality, which reflects status and rights of activities in their social network, is one of the most important metrics in network analysis. There are three common measures of centrality: degree centrality, betweenness centrality and closeness centrality [11]. In collaborative networks, if there is a direct connection between an actor and others, the actor is in the central position with greater rights. Degree centrality is equal to the number

Table 1 Ten representative Journals in Chinese psychiatry field

\begin{tabular}{ll}
\hline Journal title & Number of papers \\
\hline Journal of Clinical Mental Psychiatry & 2805 \\
Chinese Journal of Nervous and Mental Disease & 2598 \\
Journal of Neuroscience & 2147 \\
Journal of Clinical Psychosomatic Diseases & 1979 \\
Journal of Psychiatry & 1756 \\
Shang Hai Archives of Psychiatry & 1449 \\
Si Chuan Mental Health & 1442 \\
Chinese Journal of Psychiatry & 1098 \\
Journal of Neurology and Neurorehabilitation & 732 \\
Journal of International Psychiatry & 618 \\
\hline
\end{tabular}

of nodes that connect with a central node. If an author/ institution/region has the highest degree centrality, it is considered a central author/institution/region in the collaboration network [12]. In collaborative networks, if an actor is between two points, that actor is in the important posiition. Betweenness centrality is the number of shortest paths that pass through a given node [13]. In our study, having the highest betweenness centrality would indicate that the author/institution/region possesses and controls a great deal of resources for research. Finally, in collaborative networks, if all paths between an actor and others respectively are the shortest, this actor is in the core position. Closeness centrality of a given node is equal to the reciprocal of the total distance from this node to all other nodes. Thus, the closer a node is to all other nodes, the higher its closeness centrality. Having the lowest closeness centrality indicates that the author/institution/region is at the core of the entire network.

In Core-Periphery structure analysis, the network is divided into two arears-core area and periphery area. The nodes which are in the core area are in the important position.

Hierarchical clustering, which creates a hierarchy of clusters that can be represented by a dendrogram, has been used to extract subgroups from the co-authorship network in many studies. In this tree structure, the root of the tree consists of a single cluster containing all authors, while the leaves correspond to specific individuals.

SNA was used to analyze the collaboration structure of Chinese psychiatry research. We then used the UCINET program and Netdraw to produce a visual representation of the network. In addition, the software for frequency analysis was SATI (Statistical Analysis toolkit For Informetrics) published by Zhe Jiang University in China (Http://sati.liuqiyuan.com/\#sati).

Firstly, we imported all the data into SATI and produced the co-occurrence matrix. Then, the information of the matrix was imported into UCINET to analysis of centrality, hierarchical clustering and core-periphery structure. Finally, we imported them to Netdraw to the analysis of visualization and formed the mapping knowledge domain.

In order to elucidate the main co-authorship structure of the network, we selected the 100 most prolific authors, the top 100 institutions and 32 regions from 2003 to 2012. This threshold resulted in the top 100 prolific authors who must have published 32 co-authorship papers to be included (two authors who have not collaborated with others were deleted, so the co-authorship map, a visualization of authors' collaboration network, includes 98 authors). The top 100 institutions appeared more than 24 times (25 institutions which have not collaborated with others were deleted, so the collaborative 
map, a visualization of institutions' collaboration network, includes 75 institutions) and there were 32 regions (there were no collaborations from Tibet, so it was excluded, leaving us with 31 regions).

\section{Results}

We retrieved 16,224 papers spanning 2003-2012 from the ten psychiatry journals. Among those, 13,669 were co-authored. The percentage of co-authored papers rose from 78 to $80 \%$ over the last 10 years, suggesting an increase in scientific collaboration in the Chinese psychiatry field.

\section{Analysis on collaboration at the micro (authors) level}

Of all the publications from the Chinese psychiatry field in 2003 to 2012, more than $80 \%$ were published by two or more collaborators and the output of achievements in scientific research by way of collaboration was consistent with the total output. This suggested that most published research was collaborative. In this study, the map was composed of 5 independent sub-networks. The line value and the distance between two vertices represent collaborative strength, while thickness of the line represents the number of co-authorship papers. In this authors' collaboration network, the researcher working in Shanghai Mental center has the highest degree centrality of 33, indicating that with 33 collaborators, he was the most key author. In a collaboration network, betweeness centrality reflects the role that the author plays within the network. Without the author with the highest betweeness centrality, the collaborative network would be disrupted. Since the researcher working in Nanjing Medical University was the author with the highest betweeness centrality, he played an instrumental role in maintaining the cohesion of the network and thus had the power to influence collaborative relationships. In a collaborative network, the closer two authors are to each other, the more easily information is communicated and the more likely the two are to collaborate. The researcher working in Shanghai Mental health center had the lowest closeness centrality, indicating that he contributed a great deal of research and was in a central position within the entire network.

Using hierarchical clustering analysis, we divided the 98 authors into 5 sub-networks. The largest sub-network included 90 nodes and 1132 lines. In this sub-network, the average path length was 2.495 and the average clustering coefficient was 3.288 , indicating a clustering effect. A researcher, who is in this sub-network, worked in the Shanghai Mental Health Center and had the highest centrality degree mainly due to his research focusing on neuroimaging of patients with mental disorders. The researcher, who also worked in the Shanghai Mental Health Center, had the second highest centrality degree mainly due to his research focusing on the neuropathology of mental disorders. The researcher, with the third highest centrality degree, mainly researched treatments for schizophrenia. The second sub-network included two researchers, who both worked in the Tian shui Mental Hospital of Gansu and researched pharmacological treatments of depression. The third sub-network included the researcher from the Fourth Hospital of Linyi in Shandong and the researcher from the Linyi Mental Health Center of Shandong, and their research focused on the classification of depression. The fourth sub-network included the researcher from Anhui Medical University and the researcher from The Fourth People's Hospital of Hefei in Anhui, and they researched the neurophysiology of mental

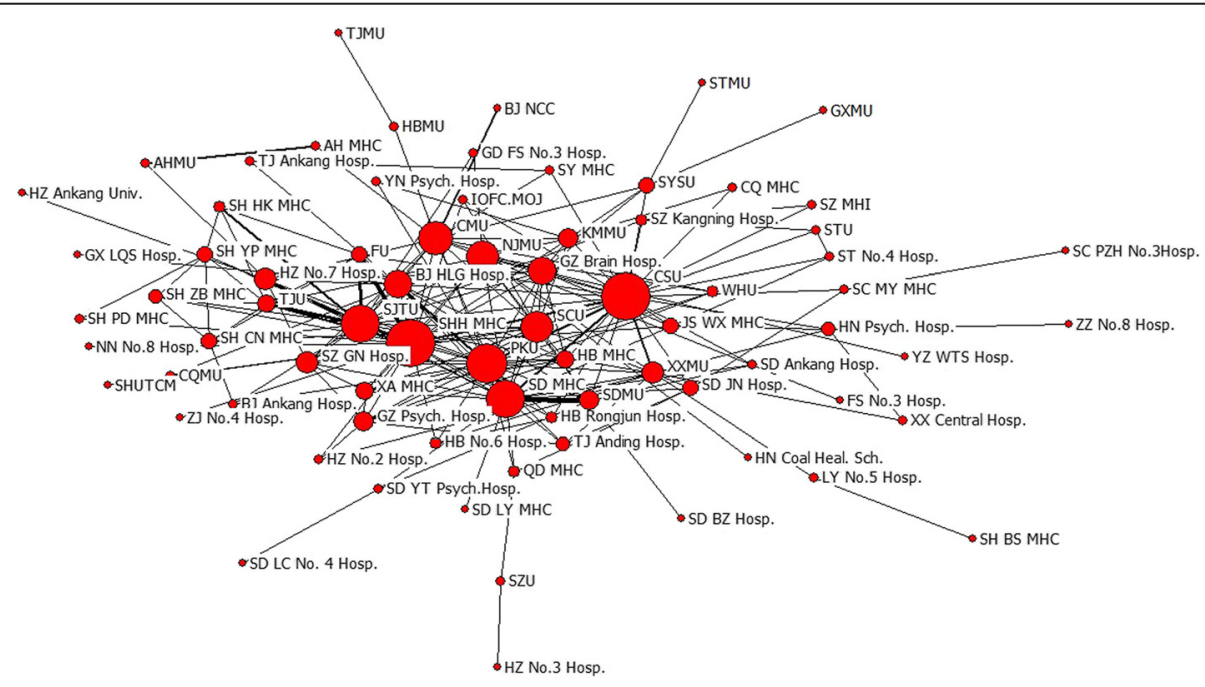

Fig. 1 The structure map of the institutional collaboration network on Chinese psychiatry research (The geographic name and the name of university adopted the official abbreviation, see Table 2) 
Table 2 The full names and acronyms of institutions

\begin{tabular}{|c|c|c|c|}
\hline Name of Institution & Acronyms & Name of Institution & Acronyms \\
\hline Anhui Medical University & $\mathrm{AHMU}$ & $\begin{array}{l}\text { Shandong Jining Municipal Hospital for Mental } \\
\text { Disease Prevention and Control }\end{array}$ & SD JN Hosp. \\
\hline Anhui Mental Health Center & $\mathrm{AH} M H C$ & Shandong Liaocheng No. 4 People's Hospital & SD LC No. 4 Hosp. \\
\hline Beijing Ankang Hospital & BJ Ankang Hosp. & Shandong Linyi Mental Health Center & SD LY MHC \\
\hline Beijing Huilongguan Hospital & BJ HLG Hosp. & Shandong Mental Health Center & SD MHC \\
\hline Beijing Neurology Consultation Center & BJ NCC & Shandong Qingdao Mental Health Center & QD MHC \\
\hline Capital Medical University & CMU & Shandong University & SDMU \\
\hline Central South University & CSU & Shandong Yantai Psychological Convalescent Hospital & SD YT Psych.Hosp. \\
\hline Chongqing Medical University & CQMU & Shanghai Baoshan District Mental Health Center & SH BS MHC \\
\hline Chongqing Mental Health Center & CQ MHC & Shanghai Changning District Mental Health Center & SH CN MHC \\
\hline Foshan No.3 People's Hospital & FS No.3 Hosp. & Shanghai Hongkou District Mental Health Center & SH HK MHC \\
\hline Fudan University & FU & Shanghai Jiao Tong University & SJTU \\
\hline Guangdong Foshan No.3 People's Hospital & GD FS No.3 Hosp. & Shanghai Mental Health Center & $\mathrm{SHH} \mathrm{MHC}$ \\
\hline Guangdong Shantou No.4 People's Hospital & ST No.4 Hosp. & Shanghai Pudong New District Mental Health Center & SH PD MHC \\
\hline Guangxi Longquanshan Hospital & GX LQS Hosp. & Shanghai University of Traditional Chinese Medicine & SHUTCM \\
\hline Guangxi Medical University & GXMU & Shanghai YangPu District Mental Health Center & SH YP MHC \\
\hline Guangxi Nanning No.8 People's Hospital & NN No.8 Hosp. & Shanghai Zhabei District Mental Health Center & SH ZB MHC \\
\hline Guangzhou Brain Hospital & GZ Brain Hosp. & Shantou University & STU \\
\hline Guangzhou Psychiatric Hospital & GZ Psych. Hosp. & Shenzhen Kangning Hospital & SZ Kangning Hosp. \\
\hline Hangzhou No.7 People's Hospital & HZ No.7 Hosp. & Shenzhen Mental Health Institute & $\mathrm{SZ} \mathrm{MHI}$ \\
\hline Hangzhou Police Station Ankang University & HZ Ankang Univ. & Sichuan Mianyang Mental Health Center & SC MY MHC \\
\hline Hebei Medical University & $\mathrm{HBMU}$ & Sichuan Panzhihua No.3 People's Hospital & SC PZH No.3Hosp. \\
\hline Hebei Mental Health Center & $\mathrm{HB} M H C$ & Sichuan University & SCU \\
\hline Hebei No.6 People's Hospital & HB No.6 Hosp. & Southern Medical University & STMU \\
\hline Hebei Rongjun Hospital & HB Rongjun Hosp. & Sun Yat-sen University & SYSU \\
\hline Henan Coal Health School & HN Coal Heal. Sch. & Suzhou Guangji Hospital & SZ GN Hosp. \\
\hline Henan Psychiatric Hospital & HN Psych. Hosp. & Suzhou University & SZU \\
\hline Huizhou No.2 People's Hospital & HZ No.2 Hosp. & Tianjin Anding Hospital & TJ Anding Hosp. \\
\hline $\begin{array}{l}\text { Institute of Forensic Science.Ministry of } \\
\text { Justice P.R. China }\end{array}$ & IOFC.MOJ & Tianjin Ankang Hospital & TJ Ankang Hosp. \\
\hline Jiangsu Wuxi Mental Health Center & JS WX MHC & Tianjin Medical University & TJMU \\
\hline Jiangsu Yangzhou Wutaishan Hospital & YZ WTS Hosp. & Tongji University & TJU \\
\hline Jiangsu Zhenjiang No.4 People's Hospital & ZJ No.4 Hosp. & Wuhan University & WHU \\
\hline Kunming Medical University & KMMU & Xian Mental Health Center & XA MHC \\
\hline Liaoning Shenyang Mental Health Center & SY MHC & Xinxiang Central Hospital & XX Central Hosp. \\
\hline Luoyang No.5 People's Hospital & LY No.5 Hosp. & Xinxiang Medical University & XXMU \\
\hline Nanjing Medical University & NJMU & Yunnan Psychiatric Hospital & YN Psych. Hosp. \\
\hline Peking University & PKU & Zhejiang Huzhou No.3 People's Hospital & HZ No.3 Hosp. \\
\hline Shandong Ankang Hospital & SD Ankang Hosp. & Zhengzhou No.8 People's Hospital & ZZ No.8 Hosp. \\
\hline Shandong Binzhou People's Hospital & SD BZ Hosp. & & \\
\hline
\end{tabular}

disorders. Finally, the fifth sub-network included two researchers from Xuanwu Hospital in Beijing, where they researched neurology.
Analysis on collaboration at the meso (institutions) level Papers with multi-institutional collaborators accounted for more than half of all papers. Universities, research 
institutions and hospitals were the main institutions conducting research in this field. As collaborations among institutions increased, the total output in terms of scientific publications also increased from 2003 to 2012. In this study, there were 7,672 papers from SCI which demonstrated inter-institution collaboration from 2003 to 2012. This number increased from 418 in 2003 to 587 in 2012. These papers included 8,382 institutions that appeared a total of 32,410 times. The largest collaboration in our sample involved 20 institutions. We formed a map visualizing the structure of institutions' collaboration network in the field of psychiatry during 2003 to 2012 (Fig. 1), the abbreviations of the geographic name and the name of university were listed in Table 2. The size of the node represents centrality in collaborative network. Shandong Mental Health Center and Mental Health Center of the Second Xiangya Hospital in Central South University had the highest degree centrality and betweenness centrality, and the lowest closeness centrality (Table 3). This indicated that these institutions were highly collaborative. The distance and thickness of the line between two nodes represents collaborative strength and the number of collaborative papers, respectively. We found that Shandong Mental Health Center, Mental Health Center of the second Xiangya Hospital in Central South University and Mental Health Center of Peking University were in the center of the collaborative network and thus played an influential role in the development of psychiatry. In contrast, The eighth People's Hospital of Zhenzhou and The Fourth People's Hospital of Liaocheng in Shandong were on the edge of the collaborative network. Compared to that of those institutions in the center, the scientific research strength of institutions on the edge were slightly weaker. Further, collaborations among the institutions in the center reflect 'the center of the obvious effect' in the process of coauthorship while the institutions on the edge collaborated more loosely. Analyzing Core-Periphery was a quantitative way to study networks. We applied this method to the collaboration network and found that the 'correlative value of collaborative network' was 0.698 (UCINET 6.0). A welldelineated Core-Periphery structure appeared (Fig. 2), indicating that the collaborative network was regional, whereby research institutions in close geographical proximity were more likely to collaborate, the abbreviations of the geographic name and the name of university were listed in Table 2. Shandong Mental Health Center and Shandong University, with the greatest number of collaborations, had a close collaborative research relationship.

\section{Analysis on collaboration at the macro (regions) level}

From 2003 to 2012, the most productive regions were Shanghai, Beijing and Shandong (Fig. 3), with 2,154 psychiatric research papers originated from Shanghai, making up $13.07 \%$ of all such papers. Using the top 31 most productive regions in China to construct the map of scientific collaboration (Fig. 4), the network included 31 nodes and 226 lines. The average path length was 1.858 and the average clustering coefficient was 5.363, indicating a pronounced clustering effect. We applied Core-Periphery analysis and calculated the correlative value of the collaborative network to be 0.904 (UCINET 6.0). Once again, a well-delineated Core-Periphery structure appeared (Fig. 5).

We analyzed the effect of scientific collaboration on regions' scientific output by correlating nodes to their corresponding scientific research achievements. For each of the 31 regions, we examined the number of ties and number of papers produced (Table 4). We found that the quantity of international collaborations correlates with research output. This suggested that international scientific collaboration greatly influenced scientific output in this field, whereby countries that collaborated frequently had a greater research output.

A centrality analysis (Table 5) revealed that Beijing, with the highest degree centrality of 120 , highest betweenness

Table 3 Top 10 institutions on centrality measures in collaborative network

\begin{tabular}{llllll}
\hline Degree & Score & Betweeness & Score & Closeness & Score \\
\hline Shanghai Mental Health Center & 106 & Central South University & 1450 & Central South University \\
Shanghai Jiaotong University & 100 & Shanghai Mental Health Center & 1094 & Shanghai Mental Health Center \\
Central South University & 98 & Shandong Mental Health Center & 776 & Shandong Mental Health Center & 136 \\
Shandong Mental Health Center & 92 & Capital Medical University & 764 & Shanghai Jiaotong University \\
Fudan University & 65 & Nanjing Medical University & 747 & Peking University \\
Peking University & 58 & Peking University & 568 & Capital Medical University \\
Shandong University & 58 & Shanghai Jiaotong University & 555 & Nanjing Medical University \\
Tongji University & 42 & Xinxiang Medical University & 524 & Guangzhou Brain Hospital \\
Capital Medical University & 40 & Beijing Huilongguan Hispital & 376 & Sichuan University \\
Xinxiang Medical University & 33 & Sun Yat-sen University & 294 & Beijing Huilongguan Hispital & 146 \\
\hline
\end{tabular}




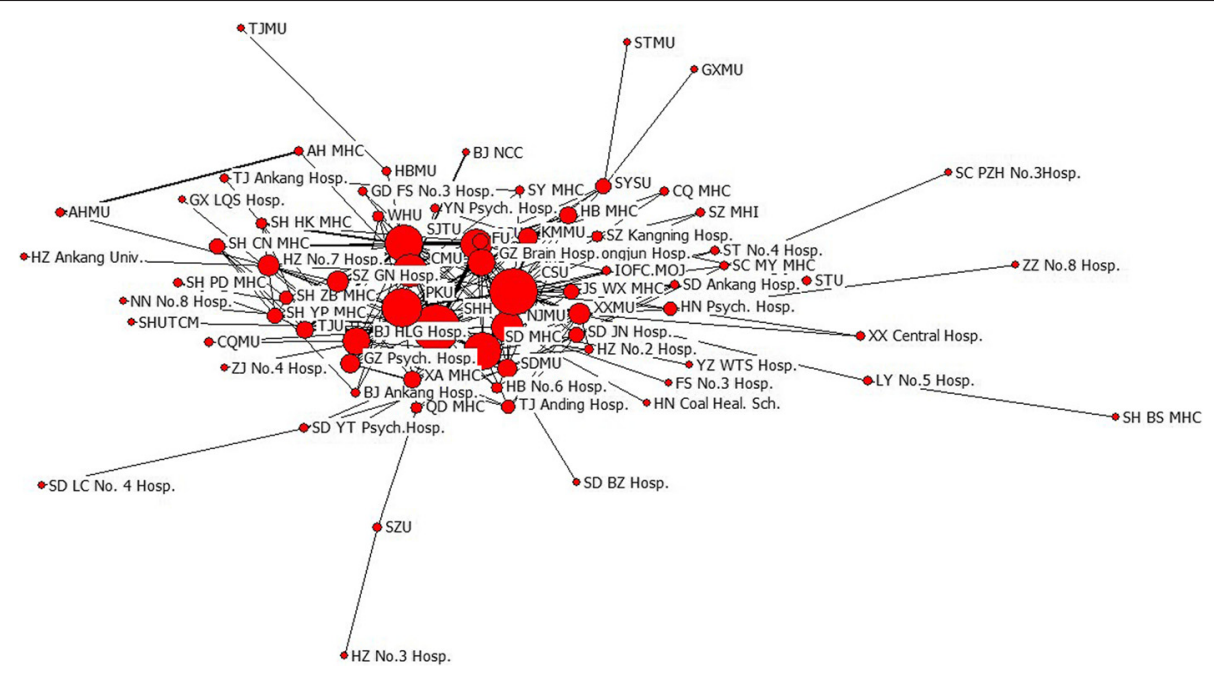

Fig. 2 The core-periphery structure map of the institutional collaboration network on Chinese psychiatry research (The geographic name and the name of university adopted the official abbreviation, see Table 2)

centrality of 177 , and lowest closeness degree of 90 , was the center of the scientific collaboration network of China's psychiatry field. Beijing's extensive research output made it a major producer of international publications.

\section{Discussion}

As China's economy has grown and social pressures have increased, the prevalence of mental disorder has also grown dramatically. Because of the diversity and complexity of diseases this encompasses, scientific collaboration is indispensable if progress is to be made in the treatment of mental disorder. Although several studies have shown that collaboration has increased at the level of authors, institutions and regions [14-16], few have reported this phenomenon within the psychiatry field. This study retrieved bibliographic data of Chinese psychiatry research from 2003 to 2012 from CNKI and WanFang Database. We constructed and analyzed the structure of scientific collaboration at the micro (authors), meso (institutions) and macro (regions) levels based on SNA and found that scientific collaboration was correlated with this field's development.

The authors who had the highest centrality were the central authors of the whole network, suggesting that they heavily influenced research in the Chinese

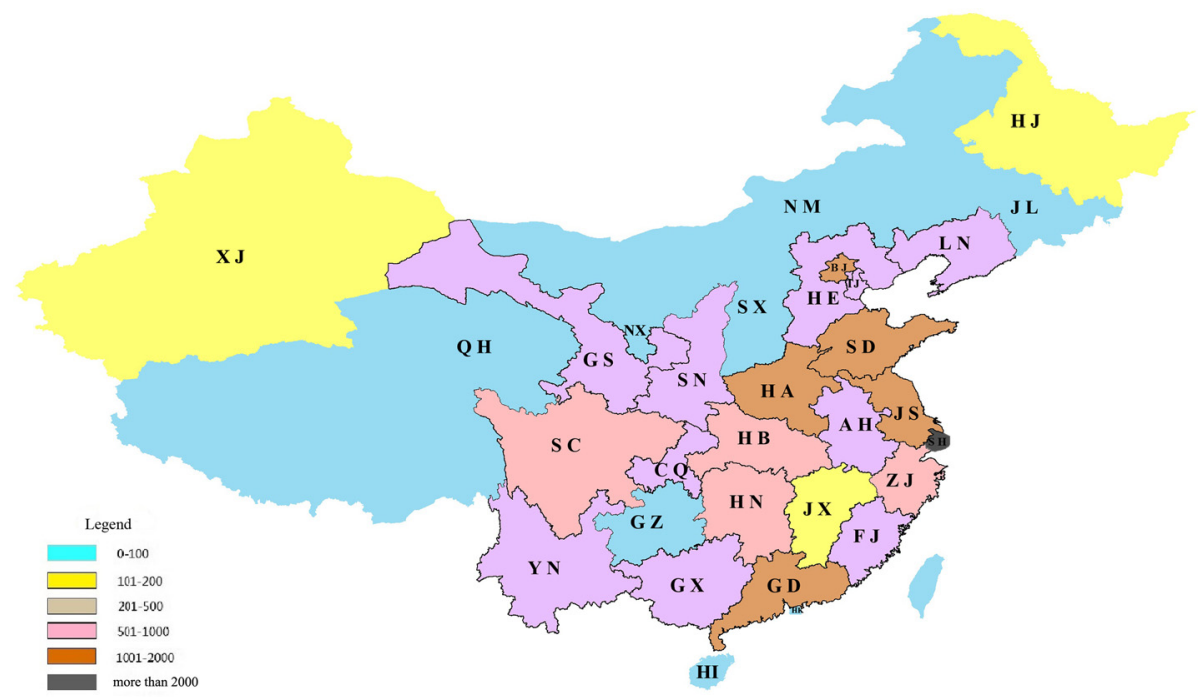

Fig. 3 Regions distribution of Chinese psychiatric papers (The geographic name adopted the official abbreviation, see Table 4) 


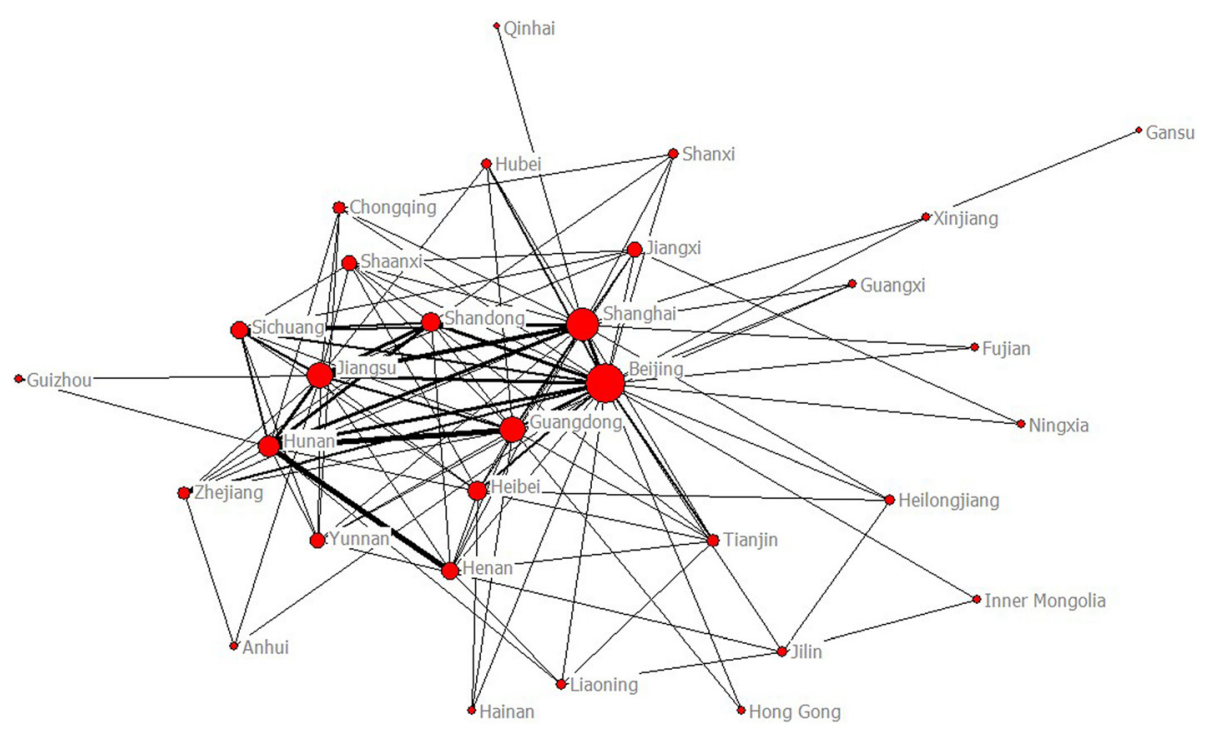

Fig. 4 The structure map of collaboration network among regions on Chinese psychiatry research

psychiatry field and were thus leader of the field. In the era of a knowledge-based economy, as the most important economic factors, intellectual resources become more obviously valuable. Thus, scientific collaboration played a large role in the emergence of a subject leader.

The institutions which had the highest centrality were the center of multi-institutional collaboration in the Chinese psychiatry field possessing and controlling a great deal of resources for research. From the analysis on Core-Periphery structure, collaborating academic institutions obviously demonstrated 'center effect', while those collaborating with famous institutions demonstrated 'elite institutions assembling'. In other words, the phenomenon that institutional collaborations was within the same country showed geographical characteristics. Thus, the other research institutions need to collaborate with institutions which collaborated closely to strive for the more scientific research resource.

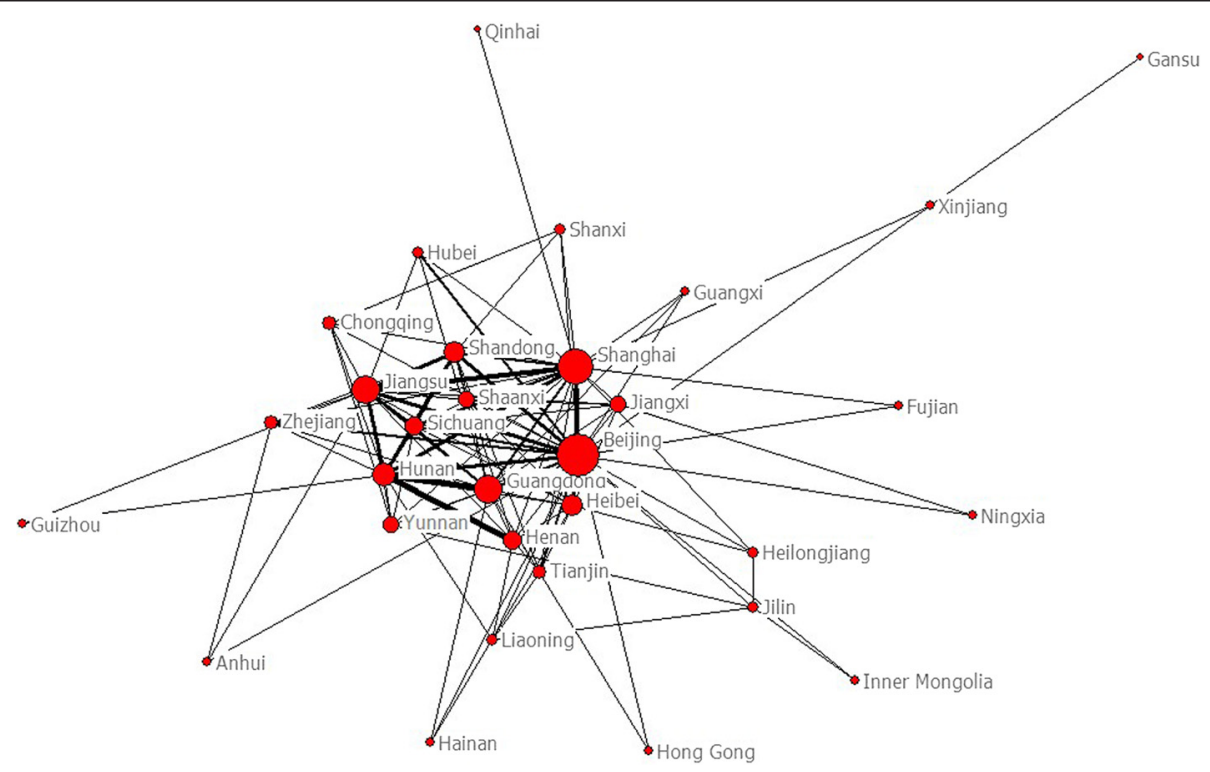

Fig. 5 The core-periphery structure map of collaboration network among regions on Chinese psychiatry research 
Table 4 The relation between scientific collaboration and papers

\begin{tabular}{|c|c|c|c|c|}
\hline \multicolumn{2}{|c|}{ Collaboration } & \multirow[t]{2}{*}{ Regions } & \multicolumn{2}{|c|}{ Production } \\
\hline Ranks & Ties & & Papers & Ranks \\
\hline 1 & 27 & Beijing(BJ) & 1214 & 6 \\
\hline 2 & 21 & Shanghai(SH) & 2154 & 1 \\
\hline 3 & 17 & Guangdong(GD) & 1495 & 4 \\
\hline 4 & 16 & Jiangsu(JS) & 1688 & 3 \\
\hline 5 & 13 & Hunan(HN) & 584 & 8 \\
\hline 6 & 12 & Hebei(HE) & 303 & 15 \\
\hline 7 & 11 & Shandong(SD) & 1754 & 2 \\
\hline 8 & 10 & Sichuan(SC) & 555 & 9 \\
\hline 9 & 9 & Henan(HA) & 1248 & 5 \\
\hline 10 & 8 & Yunnan(YN) & 368 & 12 \\
\hline 11 & 8 & Shaanxi(SN) & 284 & 16 \\
\hline 12 & 8 & Jiangxi(JX) & 140 & 22 \\
\hline 13 & 7 & Zhejiang(ZJ) & 620 & 7 \\
\hline 14 & 7 & Tianjing(TJ) & 360 & 13 \\
\hline 15 & 6 & Chongqing(CQ) & 270 & 17 \\
\hline 16 & 5 & Liaoning(LN) & 305 & 14 \\
\hline 17 & 5 & Jilin(JL) & 77 & 24 \\
\hline 18 & 4 & Hubei(HB) & 529 & 10 \\
\hline 19 & 4 & Heilongjiang(HJ) & 177 & 21 \\
\hline 20 & 4 & Shanxi(SX) & 76 & 25 \\
\hline 21 & 3 & Anhui(AH) & 369 & 11 \\
\hline 22 & 3 & Guangxi(GX) & 267 & 18 \\
\hline 23 & 3 & Xinjiang(XJ) & 107 & 23 \\
\hline 24 & 3 & Hainan(HI) & 21 & 29 \\
\hline 25 & 2 & Fujian(FJ) & 245 & 19 \\
\hline 26 & 2 & Ningxia(NX) & 54 & 26 \\
\hline 27 & 2 & Guizhou(GZ) & 53 & 27 \\
\hline 28 & 2 & Neimenggu(NM) & 42 & 28 \\
\hline 29 & 2 & HongKong(HK) & 10 & 31 \\
\hline 30 & 1 & Gansu(GS) & 237 & 20 \\
\hline 31 & 1 & Qinghai(QH) & 21 & 30 \\
\hline
\end{tabular}

At a regional level, Beijing had the highest centrality and was thus in the most central position. Our analysis suggested that regional scientific collaboration was positively correlated with total output in terms of scientific research. From the analysis on Core-Periphery structure, developed cities such as Beijing and Shanghai collaborated closely. China's rapid economic development, which has encouraged collaborative behavior, has contributed to increases in research output. Higher-income regions prefer to collaborate with each other while lower-income regions prefer to collaborate with higherincome regions in order to increase the quality of their research.
Table 5 Top 10 regions of China on centrality measures in collaborative network

\begin{tabular}{llllll}
\hline Degree & Score & Betweeness & Score & Closeness & Score \\
\hline Beijing & 120 & Beijing & 177 & Beijing & 33 \\
Hunan & 112 & Shanghai & 83 & Shanghai & 39 \\
Jiangsu & 86 & Jiangsu & 29 & Guangdong & 44 \\
Shanghai & 81 & Xinjiang & 29 & Jiangsu & 45 \\
Guangdong & 60 & Guangdong & 26 & Hunan & 48 \\
Shandong & 52 & Hunan & 18 & Hebei & 49 \\
Henan & 38 & Hebei & 8 & Shandong & 50 \\
Sichuan & 30 & Shandong & 4 & Sichuan & 51 \\
Hebei & 25 & Jiangxi & 3 & Henan & 52 \\
Tianjing & 17 & Yunnan & 3 & Yunnan & 53 \\
\hline
\end{tabular}

\section{Conclusion}

This study described the collaborative behaviors of research in the Chinese psychiatry field at the micro (authors), meso (institutions) and macro (regions) levels. Based on the centralities of the author ranking, academic leaders will be selected more easily. Furthermore, studying these collaborations not only can help researchers to master the forefront of this field but also provide scientific evidences and suggestions for policymakers to guide and manage the Chinese psychiatry field in the future.

\section{Abbreviations}

$\mathrm{AH}$, Anhui; $\mathrm{AH}$ MHC, Anhui Mental Health Center; AHMU, Anhui Medical University; BJ, Beijing; BJ Ankang Hosp., Beijing Ankang Hospital; BJ, HLG Hosp., Beijing Huilongguan Hospital; BJ NCC, Beijing Neurology Consultation Center; CMU, Capital Medical University; CNKI, China National Knowledge Infrastructure; CQ, Chongqing; CQ MHC, Chongqing Mental Health Center; CQMU, Chongqing Medical University; CSU, Central South University; FJ, Fujian; FS No.3 Hosp., Foshan No.3 People's Hospital; FU, Fudan University; GD, Guangdong; GD FS No.3 Hosp., Guangdong Foshan No.3 People's Hospital; GS, Gansu; GX, Guangxi; GX LQS Hosp., Guangxi Longquanshan Hospital; GXMU, Guangxi Medical University; GZ, Guizhou; GZ Brain Hosp., Guangzhou Brain Hospital; GZ Psych. Hosp., Guangzhou Psychiatric Hospital; HA, Henan; HB, Hubei; HB MHC, Hebei Mental Health Center; HB No.6 Hosp., Hebei No.6 People's Hospital; HB Rongjun Hosp., Hebei Rongjun Hospital; HBMU, Hebei Medical University; HE, Hebei; HI, Hainan; HJ, Heilongjiang; HK, HongKong; HN, Hunan; HN Coal Heal. Sch., Henan Coal Health School; HN Psych. Hosp., Henan Psychiatric Hospital; HZ Ankang Univ., Hangzhou Police Station Ankang University; HZ No.2 Hosp., Huizhou No.2 People's Hospital; HZ No.7 Hosp., Hangzhou No.7 People's Hospital; IOFC.MOJ, Institute of Forensic Science.Ministry of Justice P.R.China; JL, Jilin; JS, Jiangsu; JS WX MHC, Jiangsu Wuxi Mental Health Center; JX, Jiangxi; KMMU, Kunming Medical University; LN, Liaoning; LY No.5 Hosp., Luoyang No.5 People's Hospital; NJMU, Nanjing Medical University; NM, Neimenggu; NN No.8 Hosp., Guangxi Nanning No.8 People's Hospital; NX, Ningxia; PKU, Peking University; QH, Qinghai; SATI, Statistical Analysis Toolkit For Informetrics; SC, Sichuan; SD, Shandong; SD Ankang Hosp., Shandong Ankang Hospital; SD BZ Hosp., Shandong Binzhou People's Hospital; SH, Shanghai; SN, Shaanxi; SNA, Social Network Analysis; ST No.4 Hosp., Guangdong Shantou No.4 People's Hospital; SX, Shanxi; SY MHC, Liaoning Shenyang Mental Health Center; TJ, Tianjing; WHO, World Health Organization; XJ, Xinjiang; YN, Yunnan; YZ WTS Hosp., Jiangsu Yangzhou Wutaishan Hospital; ZJ, Zhejiang; ZJ No.4 Hosp., Jiangsu Zhenjiang No.4 People's Hospital. 


\section{Acknowledgements}

The authors would like to express gratitude to the participants for their time and willingness to participate in this study.

\section{Funding}

The research reported in this paper is done as part of "Analysis on Scientific Collaboration and Trends Prediction of Research Fronts in Psychiatry Field (No. 71503152)", which both is supported by National Natural Science Foundation of China.

\section{Authors' contributions}

YW conceived of the study and carried out the medical studies and drafted the manuscript. XJ conceived of the study, participated in the study's design and coordination. Both authors read and approved the final manuscript.

\section{Competing interests}

The authors declare that they have no competing interests.

\section{Consent to publish}

Not applicable.

\section{Ethics approval and consent to participate}

Not applicable.

\section{Author details}

'School of Humanities and Social Sciences, Shanxi Medical University, No. 56 South Xinjian Road, Taiyuan, China. ${ }^{2}$ Affiliated Tumor Hospital, Shanxi Medical University, Taiyuan 030013, China.

\section{Received: 12 December 2015 Accepted: 16 May 2016}

\section{Published online: 26 May 2016}

\section{References}

1. Geng L. Mental health human resource status and development. Chinese Hospitals. 2007:11(3):37-40.

2. Glanzel W, Schubert A. Analyzing scientific networks through co-authorship In: MOED, H. F., Glanzel W, Schmoch U(Eds), Handbook of Quantitative Science and Technology Research: The Use of Publication and Patent Statistics in Studies of S\&T Systems, Kluwer, Dordrecht, pp. 2004; $257-276$

3. Newman MEJ. The Structure of Scientific Collaboration Networks [J]. PNAS. 2001a;98(2):404-9.

4. Newman MEJ. Scientific collaboration networks.I. Network construction and fundamental results [J]. Phys Rev E. 2001b;64:016-131.

5. Newman MEJ. Scientific collaboration networks.II. Shortest paths, weighted networks, and centrality[J]. Phys Rev E. 2001c;64:016-132.

6. Kretschmer $\mathrm{H}$. Coauthorship networks of invisible colleges and institutionalized communities. Scientometrics. 1994;30(1):363-9.

7. Kretchmer H. Types of two-dimensional and three-dimensional collaboration patterns [C]. Proceedings of the Seventh Conference of the International Society for Scientometrics and Informetrics. Edited by C.A. MaciasChapula. Universidad de Colima, Clima, Mexico, 1999: 244-257.

8. Otte E, Rousseau R. Social network analysis: a powerful strategy, also for the information sciences. J Inf Sci. 2002;28(6):443-55.

9. Kretschmer $\mathrm{H}$, Aguillo I. Visibility of collaboration on the Web. Scientometrics. 2004;61(3):405-26.

10. Oliveira M, Gama J. An overview of social network analysis. Wiley Interdiscip Rev-Data Mining Knowl Discov. 2012;2:99-115.

11. Wasserman S, Faust K. Social network analysis: Methods and applications. London: Cambridge University Press; 1994

12. Liu ZY, Chen Y, Hou HY, et al. Mapping Knowledge Domains Methods and Application. Beiling: People's Publishing House; 2008.

13. Freeman LC. Centrality in Social Networks: Conceptual Clarification. Social network. 1979:1:215-39.

14. Uddin S, Hossain L, Abbasi A, Rasmussen K. Trend and efficiency analysis of co-authorship network. Scientometrics. 2012:90:687-99.

15. Kliegl R, Bates D. International collaboration in psychology is on the rise Scientometrics. 2011:87:149-58

16. Sin S-CJ. International Coauthorship and Citation Impact: A Bibliometric Study of Six LIS Journals, 1980-2008. J Am Soc Inf Sci Technol. 2011:62:1770-83.

\section{Submit your next manuscript to BioMed Central and we will help you at every step:}

- We accept pre-submission inquiries

- Our selector tool helps you to find the most relevant journal

- We provide round the clock customer support

- Convenient online submission

- Thorough peer review

- Inclusion in PubMed and all major indexing services

- Maximum visibility for your research

Submit your manuscript at www.biomedcentral.com/submit
Biomed Central 